\title{
PI3K Alpha/mTOR Inhibitor PWT33597 Mesylate
}

National Cancer Institute

\section{Source}

National Cancer Institute. PI3K Alpha/mT OR Inhibitor PWT 33597 Mesylate. NCI

Thesaurus. Code C97915.

The mesylate salt form of PWT 33597, an orally bioavailable dual inhibitor of phosphatidylinositide 3-kinase (PI3K) alpha and mammalian target of rapamycin (mTOR) kinase with potential antineoplastic activity. PI3K alpha/mT OR dual inhibitor PWT 33597 selectively inhibits both PI3K alpha kinase and mT OR kinase, which may result in tumor cell apoptosis and growth inhibition in PI3K/mT OR-overexpressing tumor cells. Activation of the PI3K/mT OR pathway promotes cell growth, survival, and resistance to chemotherapy and radiotherapy; mT OR, a serine/threonine kinase downstream of PI3K, may also be activated independent of PI3K. 